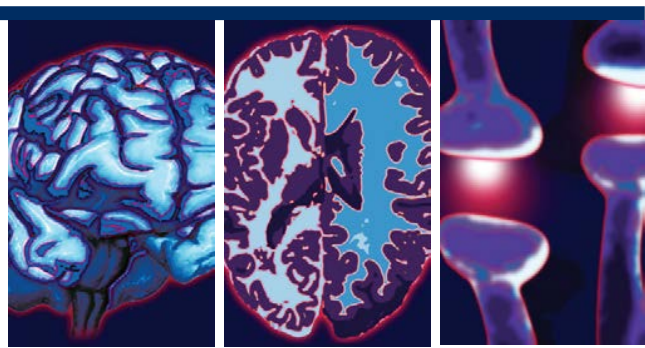

\title{
Electroconvulsive Therapy Modulates the Structural and Functional Architecture of Frontal Pole in Major Depressive Disorder
}

Jinping $\mathrm{Xu}^{1}$, Qiang Wei², Ziyun $\mathrm{Xu}^{1}$, Qingmao Hu' ${ }^{1}$ Yanghua Tian², Kai Wang², Jiaojian Wang ${ }^{3, \dagger}$

\begin{abstract}
Background:

Although electroconvulsive therapy (ECT) is the most potent treatment for severely major depressive disorder (MDD), little is known about the neural mechanism of ECT in MDD patients. The frontal pole (Fp) plays an important role in integrating social, emotional, and cognitive processes. However, the exact role of $\mathrm{Fp}$, especially at the sub-regional level, response for ECT in MDD remains largely unknown.
\end{abstract}

\section{Methods and Findings:}

We combined voxel-based morphometry and resting-state functional connectivity (RSFC) to investigate the structural and functional alterations in Fp sub-regions to explore the mechanism of ECT in 23 MDD patients before and after ECT. Structurally, we found increased gray matter volume (GMV) of the left Fp1 and left Fp2 in MDD patients after ECT. Functionally, we found decreased RSFC between the left Fp1 and right cerebellum, left fusiform gyrus, and between right Fp1 and left fusiform gyrus, as well as increased RSFC between right $\mathrm{Fp} 1$ and left angular gyrus, left cuneus, and between right $\mathrm{Fp} 2$ and left cuneus in MDD patients after ECT. Furthermore, we also found significant associations between the changes of the GMV/RSFC and the therapeutic efficacy or side effects of ECT in MDD patients.

\section{Conclusion:}

These results showed that the ECT can distinctly modulate GMV and RSFC of Fp at subregional level in MDD patients, which provide a novel view to understand the mechanism of ECT and may help us optimize the ECT procedures for improving therapeutic efficacy and reducing side effects.

\section{Keywords:}

Major depressive disorder, Electroconvulsive therapy, Frontal pole, Resting-state functional connectivity, Gray matter volume

'Institute of Biomedical and Health Engineering, Shenzhen Institutes of Advanced Technology, Chinese Academy of Sciences, Shenzhen 518055, China

${ }^{2}$ Department of Neurology, the First Hospital of Anhui Medical University, Hefei, 230022, China

${ }^{3}$ Key Laboratory for Neurolnformation of the Ministry of Education, School of Life Science and Technology, University of Electronic Science and Technology of China, Chengdu 625014, China

${ }^{\dagger}$ Author for correspondence: Dr. Jiaojian Wang, School of Life Science and Technology, University of Electronic Science and Technology of China, Chengdu 610054, P.R. China, Phone: +86-028-8320 6265; email: jiaojianwang@uestc.edu.cn 


\section{Introduction}

Major depressive disorder (MDD) is the leading global cause of disability with approximately 350 million people suffering from it in the world [1]. Despite of the availability of numerous psychopharmacological treatments, evidence indicates that only $60-70 \%$ of persons who tolerate antidepressants will respond to firstline drug therapy. Among all the antidepressant methods, the electroconvulsive therapy (ECT) was the most potent and rapid one for severely MDD patients and treatment-resistant MDD patients, leading to remission in about $50-70 \%$ of such patients [2]. However, the clinical use of ECT is accompanied by safety concerns due to the potentially long-lasting unfavorable effects on memory and other cognitive functions [3]. Indeed, cognitive changes, especially the retrograde and anterograde memory deficits, have been widely reported due to ECT [4]. Although several previous studies were performed to uncover the mechanism of the ECT in the MDD patients [5-7], the mechanism underlying the therapeutic efficacy and side effects of ECT in MDD patients is still under debate, which hinders the optimization of ECT procedures for improving therapeutic efficacy and reducing side effects.

Most previous neuroimaging studies have identified functional and structural abnormalities of the frontal pole $(\mathrm{Fp})$ in MDD patients. Functionally, it has been reported that the Fp was involved in behavioral disturbances related to introspective evaluation [8], self-relevant reflection [9], and rumination occurrence [10] in MDD patients. Corroborating the functional considerations, there are also reports about structural alterations of the Fp in MDD patients, such as reduced gray matter volume (GMV) [11,12], cortical thickness [13], and variations in the neurophysiological metabolism of receptors [14]. In addition, a single-photon emission computed tomography-based study also demonstrated cerebral blood flow reduction in the left frontopolar gyrus in MDD patients after ECT [15]. Moreover, a recently developed variant of ECT, known as focal electrically administered seizure therapy, takes the Fp as targets during treatment of MDD [16,17]. Considering such important roles of the $\mathrm{Fp}$ in the MDD patients, thus, investigations of the functional and structural alterations of the $\mathrm{Fp}$ in MDD patients after ECT provide us a novel view to understand the mechanism of ECT.
Recently, histological examination demonstrated the presence of two distinct cytoarchitectonic areas in the human Fp: area frontopolaris 1 (Fp1, lateral) and area frontopolaris 2 (Fp2, medial) [18]. Interestingly, sub-region based morphometric analysis only found a significantly smaller left $\mathrm{Fp} 2$ in the MDD patients, accompanied by a negative correlation of disease severity and duration [19]. While using independent component analysis, reduced connectivity of the left Fp1 in a network associated with attention and working memory was identified in MDD patients [20]. These discrepant results raise two questions: (1) whether the ECT can distinctly modulate structural and functional patterns of the $\mathrm{Fp}$ at sub-regional level in MDD patients; and (2) how they related to the therapeutic efficacy and side effects of ECT in MDD patients.

To answer the above questions, we combined voxel-based morphometry (VBM) and restingstate functional connectivity (RSFC) to study the structural and functional changes of $\mathrm{Fp}$ sub-regions defined using cytoarchitectonic probabilistic maps to explore the mechanism of ECT in 23 MDD patients before and after ECT. First, we compared the GMV and the RSFC patterns of the bilateral sub-regions of Fp in MDD patients before and after ECT. Next, correlation analyses were used to explore the associations between the changes of GMV/ RSFC and the changes of Hamilton Rating Scale for Depression (HRSD), Delayed Recall of Auditory Verbal Learning Test (AVLTDR), and Immediate Recall of Auditory Verbal Learning Test (AVLT-IR) scores in the MDD patients before and after ECT.

\section{Methods \\ - Participants}

We recruited patients with diagnoses of depression referred for ECT by psychiatrists from the Anhui Mental Health Center between 2012 and 2015. The diagnoses of depression were established at the basis of Diagnostic and Statistical Manual of Mental Disorders-IV criteria. Patients who showed resistance to drug therapy or a severe suicidal tendency were referred for ECT. Patients with ECT before the current course, substance dependence, and pregnancy, 
life threatening somatic disease, neurological disorders, other comorbid mental disorders or MRI-related contraindications were excluded in this study. At last, 23 patients remained for this study and all continued to take antidepression drugs during ECT administration. The detailed information of antidepression drugs can be found in the paper by Wang, et al. [21]. Demographics and clinical data of the MDD patients were shown in Table 1. All the participants were right-handed and provided written informed consent. This study was carried out in accordance with the Declaration of Helsiniki and approved by the Anhui Medical University Ethics Committee.

\section{- ECT procedures}

Patients underwent modified bi-frontal ECT in the Anhui Mental Health Center using a Thymatron System IV Integrated ECT Instrument (Somatics, Lake Bluff, IL, USA). The first three ECT administrations occurred on consecutive days, and the remaining was conducted every other day with a break of weekends until patients' symptoms remitted. The initial percent energy dial was set at the patient's age when the patient was older than 50 years (for example, $53 \%$ for a 53 -year-old patient); others were set at the value of patient's age minus five (for example, $40 \%$ for a 45 -year-old patient). Moreover, the percent energy would increase until a therapeutically satisfactory seizure was obtained. During each ECT procedure, patients were under propofol anesthesia. We administered succinylcholine and atropine to relax muscles and suppress the secretion of glands, and monitored seizure activity with electroencephalography [7]. Detailed information can be found in the paper by Wei, et al [7].

\section{- Clinical measurements}

We administered the 17-item HRSD [22] to assess the symptoms of MDD patients. They completed the scale 12-24 hours before the first ECT and 24-72 hours after the last ECT.

We also used the Auditory Verbal Learning Test (AVLT) to assess the verbal episodic memory. According to the quite difficulty of the classical AVLT, a simplified version was used in this study. At first, a list of 15 words was read to subjects with the speed one word per second. After presentation, they were immediately asked to recall as many words as possible. The AVLTIR was repeated 3 times and recalled words were recorded for 3 trials. After 10 minutes, under the condition of no presentation before, the subjects were instructed to recall the 15 words presented during AVLT-IR and AVLT-DR. The total scores for AVLT-IR (trial 1-3) and AVLT-DR were separately analyzed.

\section{- MRI data acquisition}

All patients underwent the functional MRI (fMRI) scans at the First Affiliated Hospital of Anhui Medical University. Two scans were prospectively performed at 12-24 hours before the first ECT and 24-72 hours after the last ECT for MDD patients. All participants were asked to keep their eyes closed, relaxed, awake, and not to think of anything in particular during

\begin{tabular}{|c|c|c|}
\hline Subjects & \multicolumn{2}{|c|}{ MDD } \\
\hline Number of subjects & \multicolumn{2}{|c|}{23} \\
\hline Age: mean \pm SD & \multicolumn{2}{|c|}{$38.74 \pm 11.02$} \\
\hline Gender (male : female) & \multicolumn{2}{|c|}{$11: 12$} \\
\hline Education level: mean \pm SD & \multicolumn{2}{|c|}{$8.83 \pm 3.89$} \\
\hline Durations of illness (months) & \multicolumn{2}{|c|}{$70.35 \pm 83.27$} \\
\hline Age of onset (years) & \multicolumn{2}{|c|}{$33.90 \pm 12.26$} \\
\hline Number of Treatment: mean \pm SD & \multicolumn{2}{|c|}{$7.26 \pm 2$} \\
\hline Episodes (First : Recurrence) & \multicolumn{2}{|c|}{$8: 15$} \\
\hline Family history (n patients) & \multicolumn{2}{|c|}{2} \\
\hline \multirow[t]{2}{*}{ Medication:medicationfree (n patients) } & \multicolumn{2}{|c|}{$23: 0$} \\
\hline & Before ECT & After ECT \\
\hline AVLT-IR: mean \pm SD & $19.65 \pm 8.57$ & $17.13 \pm 6.47$ \\
\hline AVLT-DR $:$ mean $\pm S D$ & $6.82 \pm 3.16$ & $3.91 \pm 3.78$ \\
\hline HRSD scores $^{b}:$ mean \pm SD & $22.22 \pm 4.74$ & $3.83 \pm 2.15$ \\
\hline \multicolumn{3}{|c|}{ 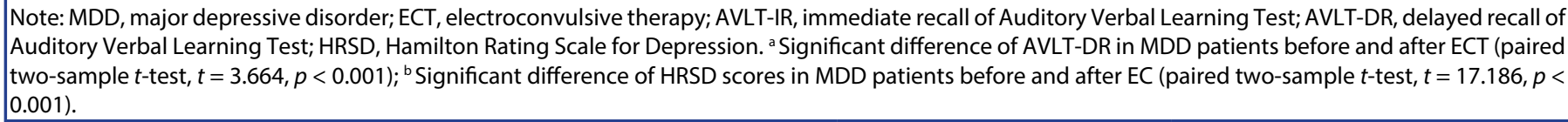 } \\
\hline
\end{tabular}


the scan. All scans were performed using a clinical 3.0 T whole body MRI scanner (Signa HDxt 3.0 T, GE Healthcare) with a standard echo planar imaging sequence. The T1-weighted anatomic images were acquired in sagittal orientation with three-dimensional inversion recovery prepared fast spoiled gradient recalled sequence (repetition time $=8.676 \mathrm{~ms}$, echo time $=3.184 \mathrm{~ms}$, inversion time $=800 \mathrm{~ms}$, flip angle $=8^{\circ}$, field of view $=256 \times 256 \mathrm{~mm}^{2}$, matrix size $=256 \times 256$, slice thickness $=1 \mathrm{~mm}$, voxel size $=$ $1 \times 1 \times 1 \mathrm{~mm}^{3}$, and sections $\left.=188\right)$. The restingstate functional images were recorded using a standard echo planar imaging sequence with the following parameters: repetition time $=2000$ $\mathrm{ms}$, echo time $=22.5 \mathrm{~ms}$, flip angle $=30^{\circ}, 240$ volumes, 33 slices, slice thickness $=4 \mathrm{~mm}$, gap thickness $=0.6 \mathrm{~mm}$, voxel size $=3.4 \times 3.4 \times 4.6$ $\mathrm{mm}^{3}$, matrix size $=64 \times 64$, and field of view $=$ $220 \times 220 \mathrm{~mm}^{2}$.

\section{- VBM analysis}

The structural MRI images were preprocessed using the standard procedure implemented in VBM8 toolbox (http://dbm.neuro.uni-jena. de/vbm.html) in SPM8 (http://www.fil.ion. ucl.ac.uk/spm). Each structural image was segmented into gray matter, white matter and cerebrospinal fluid and subsequently transformed to MNI space using DARTELnormalization. The normalized and modulated gray matter images were used for further analysis.

\section{- Resting-state fMRI data preprocessing}

Preprocessing of the resting-state fMRI data was performed with the Data Processing \& Analysis of Brain Imaging (DPABI, http://rfmri.org/ dpabi). For each participant, the preprocessing steps were: (1) the first 10 volumes of each functional time series were discarded to allow for magnetization equilibrium; (2) the slice times for the remaining 230 images were corrected and realigned to the first volume to account for head motion (subjects with head motion exceeding 3 $\mathrm{mm}$ in any dimension or $3^{\circ}$ of angular motion through the resting-state run were removed); (3) all data were spatially normalized to the MNI template and resampled to $3 \times 3 \times 3 \mathrm{~mm}^{3}$; (4) spatial smoothing was performed using a Gaussian kernel of $6 \mathrm{~mm}$ full-width at half maximum; (5) temporal band-pass filtering $(0.01-0.08 \mathrm{~Hz})$ was performed; (6) linear and quadratic trends were removed; and (7) nuisance signals, such as those from white matter, cerebrospinal fluid, the global mean signal, and six motion parameters, were regressed out. Finally, this preprocessing procedure provided a four-dimensional residual time series in a standard MNI space for each participant.

\section{- Definition of seed regions}

The region of interests (ROIs) was derived from the cytoarchitectonic probabilistic mapping of the human $\mathrm{Fp}$ in 10 human postmortem brains by calculating the maximum probability map of Fp sub-regions, which yielded Fp1 and Fp2 [18] (Figure 1). The four seed regions were resampled to $1.5 \times 1.5 \times 1.5 \mathrm{~mm}^{3}$ for VBM analysis and $3 \times$ $3 \times 3 \mathrm{~mm}^{3}$ for RSFC analysis.

\section{- Effects of ECT on the GMV of the Fp sub- regions}

For each subject, voxel-wise GMV were summed up across voxels of each of the four histological masks. Then, paired two-sample

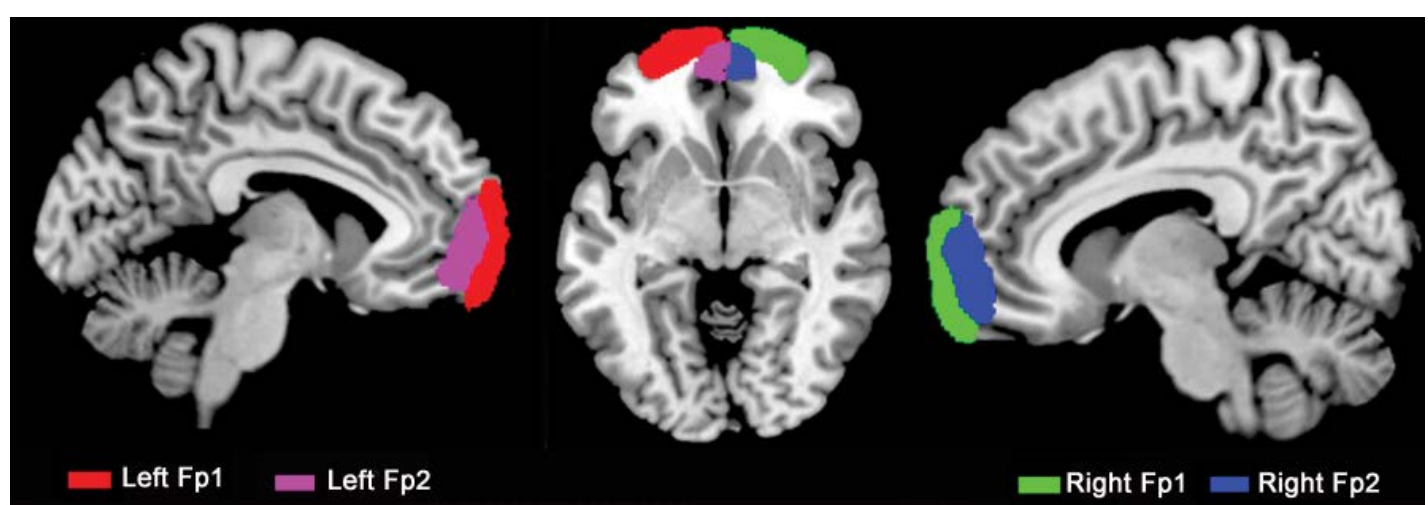

Figure 1: The maximum probability maps of the sub-regions defined within the human frontal pole (Fp). They were derived from a recent cytoarchitectonic mapping of the human Fp in 10 human postmortem brains, which yielded sub-regions Fp1 and Fp2. Abbreviations: Fp1, frontopolaris 1; Fp2, frontopolaris 2. 
Electroconvulsive Therapy Modulates the Structural and Functional Architecture of Frontal Pole in Major Depressive Disorder

$t$-tests were performed using SPSS 19.0 (SPSS, Chicago, IL, USA) to determine group difference of GMV in the patients before and after ECT. The significance was determined with $p<0.05$.

\section{- Effects of ECT on RSFC of the Fp sub- regions}

The RSFC was defined as the Pearson's correlation coefficients between the mean time series of each seed region and that of each voxel in the rest of the brain. Correlation coefficients were converted to $z$ values using Fisher's $z$ transformation to improve normality. Paired two-sample $t$-tests were performed to identify group difference of RSFC in the patients before and after ECT. The significance was determined with a cluster-level corrected threshold of $p<$ 0.05 (voxel-level $p<0.001$ using the AlphaSim method).

\section{Statistical analyses}

The statistical significant level was set at $p<0.05$. First, paired two-sample $t$-tests were performed to explore the differences in AVLT-IR, AVLTDR and HRSD scores in MDD patients before and after ECT. Then, the mean RSFC of the altered regions was calculated and paired twosample $t$-tests were performed in the patients before and after ECT.

Finally, the correlation analyses were used to explore the associations between the changed GMV, changed RSFC and the changes of the AVLT-IR, AVLT-DR and HRSD scores before and after ECT with age, gender and education as covariates.

\section{Results \\ - Effects of ECT on clinical assessments in MDD patients}

There was significantly decreased mean AVLTDR (before: $6.82 \pm 3.15$, after: $3.91 \pm 3.77$ ) in MDD patients after ECT ( $p=0.001)$, suggesting negative effects of ECT on patient's memory. There was no significant difference in AVLTIR in MDD patients after ECT compared to before $\operatorname{ECT}(p=0.178)$. In addition, there was also significant reduction of HRSD scores (before: $22.21 \pm 4.95$, after: $3.82 \pm 2.07$ ) in MDD patients after ECT $(p<0.001)$, indicating excellent therapeutic effects of ECT. The detailed information is shown in Table 1.

\section{- Effects of ECT on GMV of Fp sub-regions}

We found that the GMV of the left Fp1 and left Fp2 were significantly increased in the MDD patients after ECT (Figure 2).

\section{- Effects of ECT on RSFC of Fp sub-regions}

The distinct patterns of altered functional connectivities of Fp sub-regions were modulated by ECT in MDD patients (Figure 3). Specially, the RSFC of the left Fp1 and Cereb, the left fusiform gyrus (FG) were significantly decreased in MDD patients after ECT. The right Fp1 showed increased RSFC with the left angular gyrus (ANG) and the left cuneus (CUN), as well as decreased RSFC with the left FG in MDD patients after ECT. The right Fp2 showed increased RSFC with the left CUN in MDD patients after ECT (Table 2).

\section{- Correlation analyses}

The changes of GMV of the left Fp1 was

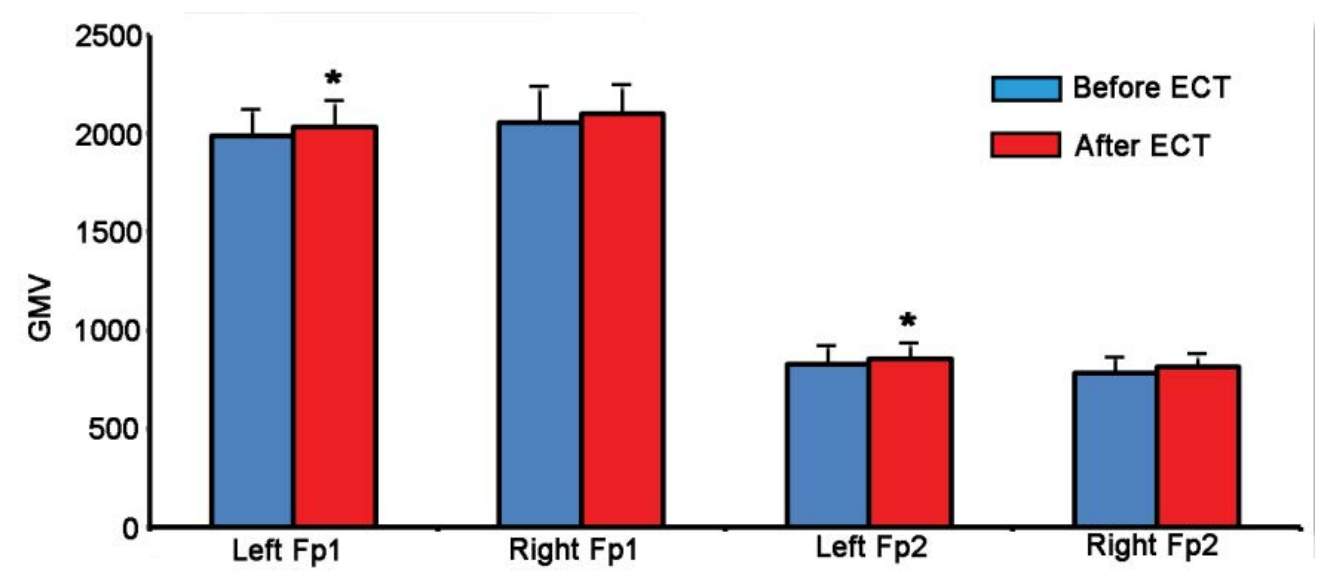

Figure 2: Effects of ECT on gray matter volume (GMV) of the Fp sub-regions in MDD patients. Paired two-sample $t$-tests were performed in MDD patients before and after ECT. *represents a significant difference between the two groups with $p<0.05$. 


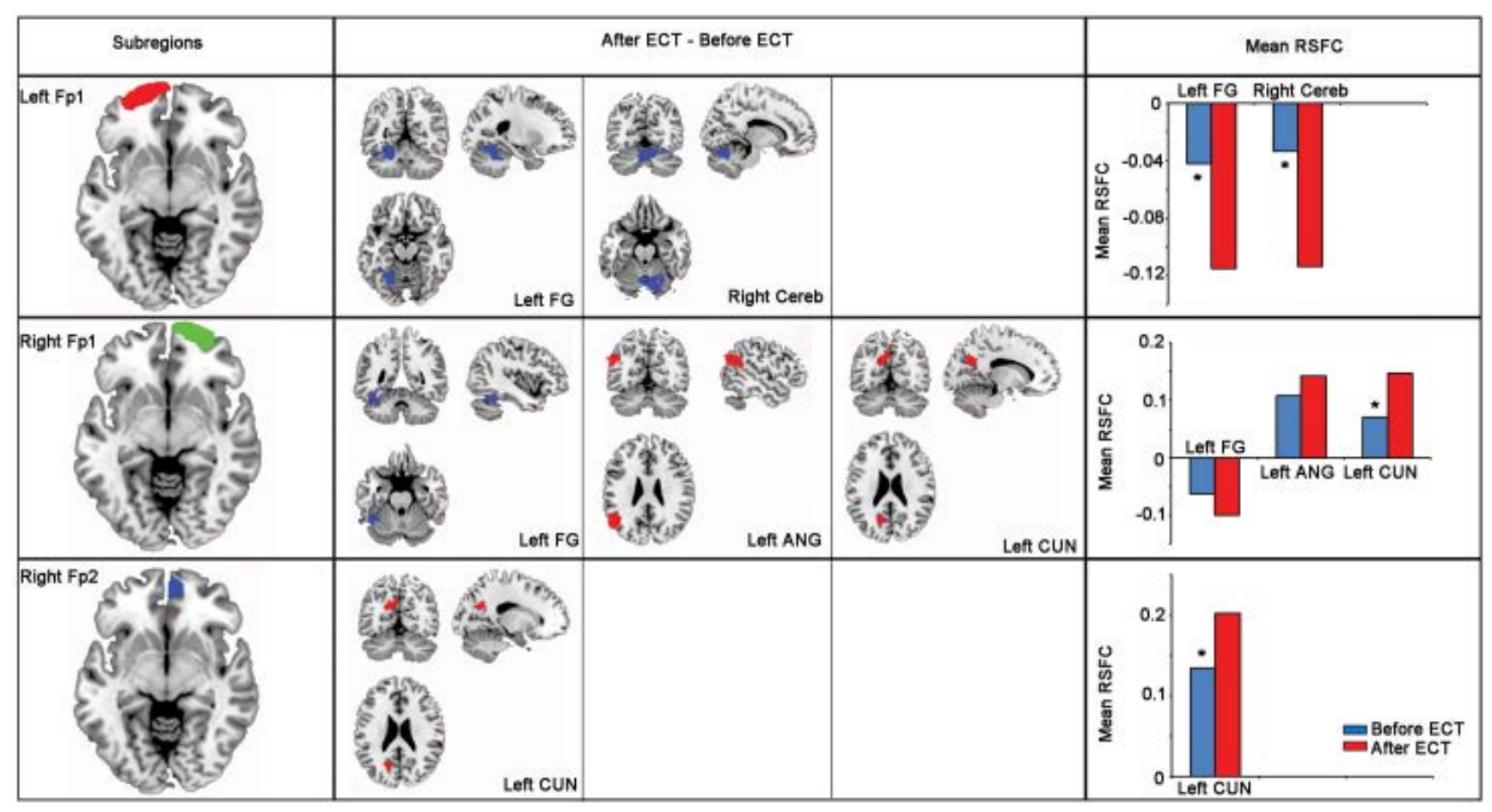

Figure 3: Effects of ECT on resting-state functional connectivity (RSFC) of the Fp sub-regions in MDD patients. Paired two-sample $t$-tests were performed in MDD patients before and after ECT. The significance was determined with a cluster-level corrected threshold of $p<0.05$ (cluster-forming threshold at voxel-level $p<0.001$ using the AlphaSim method). The first column shows the sub-regions of Fp for assessing the RSFC. The second column shows alterations of RSFC in MDD patients before and after ECT. Red and blue colors represent increased and decreased RSFC in the MDD patients after ECT, respectively. The third column shows bar plots of mean RSFC of the bilateral Fp1 and Fp2 with target regions. Paired two-sample t-tests were performed in MDD patients before and after ECT. *Represents a significant difference between the two groups with $p<0.05$. Abbreviations of brain regions are listed in Table 2.

\begin{tabular}{|c|c|c|c|c|c|c|c|}
\hline \multirow{2}{*}{$\begin{array}{l}\text { Seed regions } \\
\text { Left Fp1 }\end{array}$} & \multirow{2}{*}{$\begin{array}{l}\text { Brain regions } \\
\text { the right cerebellum }\end{array}$} & \multirow{2}{*}{\begin{tabular}{|l|} 
Abbreviations \\
Right Cereb
\end{tabular}} & \multirow{2}{*}{\begin{tabular}{|l} 
Number of voxels \\
214
\end{tabular}} & \multicolumn{3}{|c|}{ Peak MNI coordinates } & \multirow{2}{*}{\begin{tabular}{|l} 
Peak intensity \\
-7.3353
\end{tabular}} \\
\hline & & & & 12 & -66 & -21 & \\
\hline & the left fusiform gyrus & Left FG & 210 & -24 & -54 & -15 & -5.767 \\
\hline \multirow[t]{3}{*}{ Right Fp1 } & the left fusiform gyrus & Left FG & 67 & -42 & -48 & -24 & -6.486 \\
\hline & the left angular gyrus & Left ANG & 168 & -51 & -63 & 27 & 7.6936 \\
\hline & the left cuneus & Left CUN & 150 & -15 & -63 & 24 & 5.9943 \\
\hline Right Fp2 & the left cuneus & Left CUN & 89 & -18 & -63 & 24 & 5.4912 \\
\hline
\end{tabular}

significantly correlated with the changes of AVLT-DR (Figure 4A), and the changes of GMV of the left Fp2 was significantly correlated with the changes of AVLT-IR (Figure 4B). Moreover, the changes of RSFC between the right Fp1 and the left ANG was significantly correlated with the changes of HRSD scores (Figure 4C).

\section{Discussion}

To explore the mechanism of ECT, we combined VBM and RSFC to assess the structural and functional changes of $\mathrm{Fp}$ sub-regions in a group of MDD patients before and after ECT. Our results showed that ECT can selectively modulate the GMV and the RSFC patterns of
Fp sub-regions in MDD patients, suggesting lasting effects on the functional and structural architecture of the Fp. Furthermore, we found significant associations between the changes of the HRSD score and the changes of RSFC between the right Fp1 and left ANG, between the changes of the AVLT-IR and the GMV of the left Fp1, as well as between the AVLT-DR and the GMV of the right Fp2 in the MDD patients.

Using cytoarchitecture-based definition of $\mathrm{Fp}$ sub-regions, we can assess the structural and functional role of the $\mathrm{Fp}$ at the sub-regional level. Thus, we can more specifically evaluate the therapeutic efficacy and side effects of ECT to identify the exact role of Fp in MDD. 
A

B
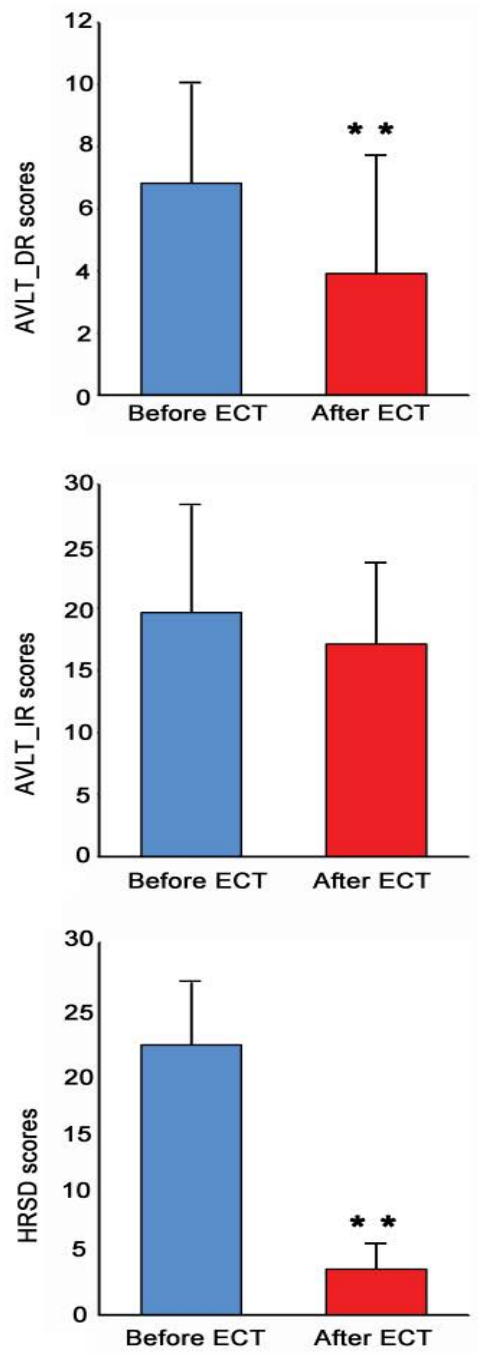
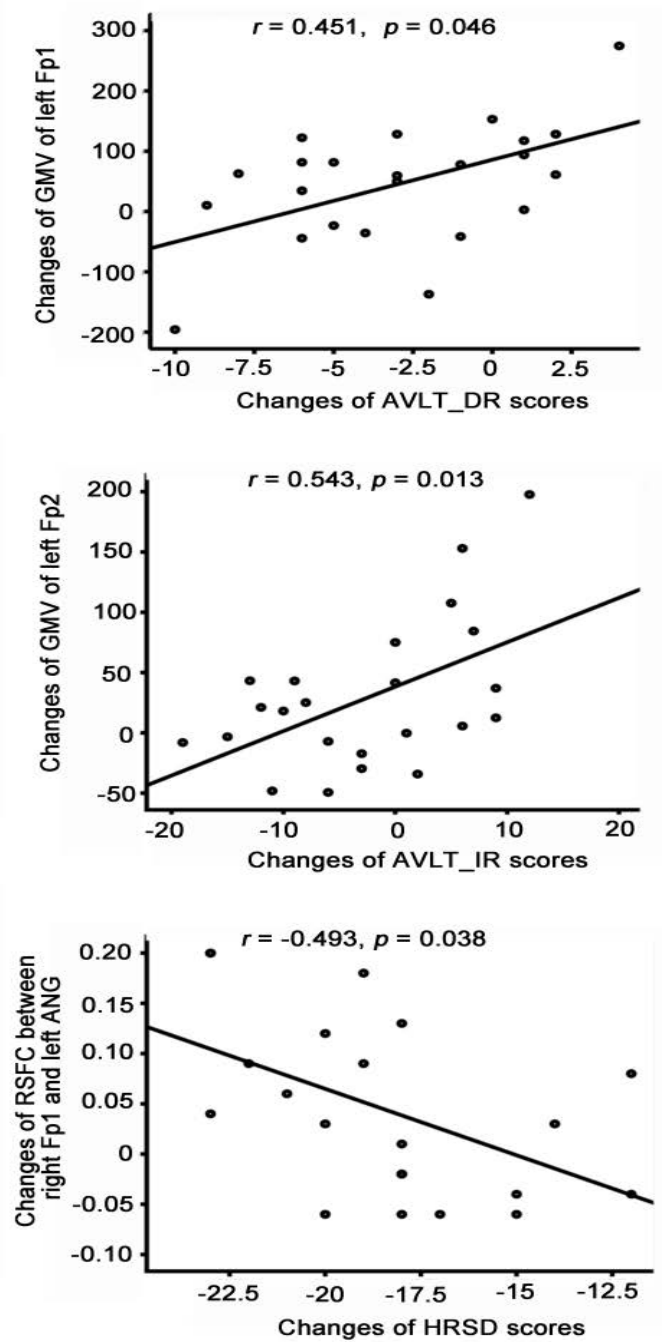

Figure 4: (A) The correlation between changes of GMV of left Fp1 and changes of AVLT-DR scores. (B) The correlation between changes of GMV of left Fp2 and changes of AVLT-IR scores. (C) The correlation between changes of HRSD scores and changes of RSFC between right Fp1 and left ANG. Abbreviations: AVLT-DR, Delayed Recall of Auditory Verbal Learning Test; AVLT-IR, Immediate Recall of Auditory Verbal Learning Test; HRSD, Hamilton Rating Scale for Depression; and ANG, the angular gyrus.

As expected, different Fp sub-regions showed different changes in structural and functional patterns in MDD patients after ECT. In addition, we found that the reductions of AVLTDR and AVLT-IR were significantly correlated with the changes of GMV of the left Fp1 and the changes of the left Fp2, respectively, whereas the reduction of HRSD scores was significantly correlated with the changes of RSFC between right Fp1 and left ANG. This finding suggested different contributions of areas Fp1 and Fp2 to the pathology of MDD, which was supported by a previous study [19]. Moreover, these different patterns described here may support the longstanding view that the therapeutic efficacy and memory deficits of ECT have distinct neurobiological substrates. Thus, it is reasonable to speculate that the therapeutic efficacy of the ECT was more related to the right Fp1, whereas the delayed and immediate memory deficits of ECT was more related to the left Fp1 and Fp2, respectively.

Structurally, we found significantly increased GMV of the left Fp1 in the MDD patients after ECT. Further analysis identified significantly decreased RSFC between the left Fp1 and the left FG and the right cerebellum (Cereb) in the MDD patients after ECT. The left Fp1 was reported to be involved in memory in many previous studies $[18,23,24]$. The left FG is a component of the 
ventral processing stream for visual information and complex feature detection [25]. Although it seems to be specifically involved in the perception of faces and recognition of face identity [26], accumulating evidence has highlighted the role of the left FG in memory, such as recency memory [27], working memory [28], long-term memory $[28,29]$, and content processing of subsequent memory [30]. Although the Cereb has long been recognized as a region which coordinates motor behavior [31], most imaging studies reported that it was involved in working memory [32,33], showing significant activation in the cerebellar posterior lobe during the working memory paradigms. Moreover, numerous neuroimaging studies have shown structural [34] or functional abnormalities [35,36] of the Cereb in MDD patients. Given the important roles of the left Fp1, left FG and right Cereb in memory, the decreased RSFC between left Fp1 and left FG, right Cereb in the MDD patients after ECT may be associated with the memory deficits after ECT. This conclusion was further supported by the correlation analysis identifying a significant association between changes of GMV of the left Fp1 and the changes of the AVLT-DR in MDD patients.

We also found significantly increased GMV of the left Fp2 in MDD patients after ECT. In MDD patients, the neurobiological alterations of the Fp were described in many previous studies, including difference in serotonin receptor densities [37] and reductions of the GMV [12]. Moreover, Bludau and his colleagues found a significantly smaller GMV of left Fp2 in MDD patients using cytoarchitectonically informed VBM [19]. Given the important roles of the left Fp2 in MDD patients, our findings of increased GMV of this sub-region may contribute to the therapeutic mechanism of ECT for MDD. However, we didn't find significant association between the changes of GMV of the left Fp2 and the changes of HRSD scores in the MDD patients after ECT, which may suggest that the left $\mathrm{Fp} 2$ is not the most effective response targets for ECT though the structural alteration was found in this sub-region. Instead, we found significant association between the changes of GMV of the left Fp2 and the changes of the AVLT-IR in the MDD patients after ECT. Therefore, the increased GMV of the left Fp2 may contribute to the memory deficits in the MDD patients.

Functionally, significantly increased RSFC between the right Fpl and left ANG was identified in the MDD patients after ECT. The left ANG was reported to be involved in MDD patients such as decreased regional cerebral blood flow [38], decreased amplitude of lowfrequency fluctuation, and increased fractional amplitude of low-frequency fluctuation [39]. In a previous study, Wei and his colleagues also identified significantly increased voxelmirrored homotopic connectivity in the ANG, which indicated that the rebalance of ANG may contribute to the therapeutic mechanism of ECT for depression [7]. Our finding was consistent with the previous study highlighting the important role of the left but not the right ANG. In a word, we speculated that the left ANG played more important roles than orther brain areas in the therapeutic mechanism of ECT for depression. Moreover, a previous study revealed that the altered nodal centrality in the left ANG was correlated with HRSD scores in the MDD patients [40]. In line with this finding, our result showed a significant association between the changes of RSFC between the right Fp1 and left ANG and the changes of HRSD scores in MDD patients. This association indicated that the RSFC between right Fp1 and left ANG may predict the therapeutic response of ECT in MDD patients.

Additionally, significantly increased RSFC between the right Fp1 and left CUN and between the right Fp2 and left CUN were identified in the MDD patients after ECT. Most previous neuroimaging studies have identified altered GMV [41,42], decreased surface area [43], reduced voxel-mirrored homotopic connectivity [44], and increased fractional amplitude of low-frequency fluctuations [45] in the left CUN in MDD patients. Moreover, the GMV in the CUN was also reported to be associated with better inhibitory control in bipolar depression patients [46]. Similarly, functional studies identified abnormal activity in the CUN during both resting state and task processing, which may lead to inhibitory deficit to generate episodic richness and imagery symptoms in MDD patients $[47,48]$. Therefore, these increased RSFC between the right Fp1 and left CUN and between the right Fp2 and left CUN may be associated with the therapeutic mechanism of ECT in MDD patients.

There are several limitations in our present study. Firstly, we assessed clinical measurements 24-72 hours after the last ECT and identified significantly decreased HRSD scores and AVLTDR scores in MDD patients. However, a recent 
meta-analytic review revealed that no significant cognitive side effects related to ECT were found after 15 days [49]. Therefore, our study could be enhanced by including a longitudinal study over a 1-month or even 1-year period to investigate lasting changes. Secondly, MDD patients take antidepressant medications at both assessment points, confounding a straightforward interpretation of ECT effects, since previous study suggested that antidepressant medications may reduce RSFC [50-60]. Although patients had failed to respond to psychotropic medication, the medication effects cannot be ruled out. Future studies of medications-naive patients that would exclude the effects of depressive medications on our findings are warranted. Finally, no normal controls are included in our study, which weaken our interpretations.

\section{Conclusion}

In the current study, we combined VBM and RSFC to uncover ECT selectively modulated the structure and function of Fp subregions in 23 MDD patients before and after ECT, suggesting lasting effects of ECT on the structural and functional architecture of the Fp. Furthermore, the significant associations between the changes of the HRSD score and the changes of functional connectivity between right Fpl and left ANG, between the changes of the AVLT-IR and the GMV of the left Fp1, as well as between the AVLT-DR and the GMV of the left Fp2 suggested that the neural indices can predict the behavioral performances of MDD patients under ECT. These findings offer us a novel view to understand the mechanism of ECT and may help us optimize the ECT procedures for improving therapeutic efficacy and reducing side effects.

\section{Funding}

This work was supported by the Science and technology innovation program of Shenzhen (JCYJ20160531184531506), the Natural Science Foundation of China (31500867, 81601187, 381671354, 61671440 and 81471117), the National Basic Research Program of China (973 program, 42015CB856400), and the Key Joint Program of National Natural Science Foundation and Guangdong Province (No.U1201257).

\section{Conflicts of Interest}

None

\section{References}

1. Oremus C, Oremus $M$, McNeely H, et al. Effects of electroconvulsive therapy on cognitive functioning in patients with depression: Protocol for a systematic review and metaanalysis. BMJ. Open 5(3), e006966 (2015).

2. Husain SS, Kevan IM, Linnell R, et al. Electroconvulsive therapy in depressive illness that has not responded to drug treatment. J. Affect. Disord 83(2-3), 121-126 (2004).

3. Kessler U, Schoeyen HK, Andreassen OA, et al. The effect of electroconvulsive therapy on neurocognitive function in treatmentresistant bipolar disorder depression. J. Clin. Psychiatry 75(11), e1306-13 (2014).

4. Trevithick L, McAllister-Williams RH, Blamire $A$, et al. Study protocol for the randomised controlled trial: Ketamine augmentation of ECT to improve outcomes in depression (ketamine-ect study). BMC. Psychiatry 15(1), 257 (2015).

5. Perrin JS, Merz S, Bennett DM, et al. Electroconvulsive therapy reduces frontal cortical connectivity in severe depressive disorder. Proc. Nat. Acad. Sci. USA 109(14), 5464-5468 (2012).

6. Abbott CC, Lemke NT, Gopal S, et al.
Electroconvulsive therapy response in major depressive disorder: A pilot functional network connectivity resting state fmri investigation. Front. Psychiatry 4(1), 10 (2013).

7. Wei Q, Tian Y, Yu Y, et al. Modulation of interhemispheric functional coordination in electroconvulsive therapy for depression. Transl. Psychiatry 4(1) (2014).

8. Christoff K, Gabrieli JDE. The frontopolar cortex and human cognition: Evidence for a rostrocaudal hierarchical organization within the human prefrontal cortex. Psychobiology 28(2), 168-186 (2000).

9. Johnson MK, Nolen-Hoeksema S, Mitchell KJ, et al. Medial cortex activity, self-reflection and depression. Soc. Cogn. Affect. Neurosci 4(4), 313-327 (2009).

10. Ray RD, Ochsner KN, Cooper JC, et al. Individual differences in trait rumination and the neural systems supporting cognitive reappraisal. Cogn. Affect. Behav. Neurosci 5(2), 156-168 (2005).

11. Koolschijn PCMP, van Haren NEM, Lensvelt-Mulders GJLM, et al. Brain volume abnormalities in major depressive disorder: $\mathrm{A}$ meta-analysis of magnetic resonance imaging studies. Human. Brain. Map 30(11), 3719-3735 (2009).
12. Grieve SM, Korgaonkar MS, Koslow SH, et al. Widespread reductions in gray matter volume in depression. Neuroimage-Clinical 3(1), 332339 (2013).

13. Canu E, Kostic M, Agosta F, et al. Brain structural abnormalities in patients with major depression with or without generalized anxiety disorder comorbidity. J. Neurology 262(5):1255-1265 (2015).

14. Gibbons AS, Scarr E, McLean C, et al. Decreased muscarinic receptor binding in the frontal cortex of bipolar disorder and major depressive disorder subjects. J. Aff. Disord 116(3), 184-191 (2009).

15. Segawa K, Azuma H, Sato K, et al. Regional cerebral blood flow changes in depression after electroconvulsive therapy. Psych. Res. Neuroimag 147(2-3), 135-143 (2006).

16. Nahas Z, Short B, Burns C, et al. A feasibility study of a new method for electrically producing seizures in man: Focal electrically administered seizure therapy [feast]. Brain. Stimul 6(3), 403-408 (2013).

17. Chahine G, Short B, Spicer K, et al. Regional cerebral blood flow changes associated with focal electrically administered seizure therapy (feast). Brain. Stimul 7(3), 483-485 (2014).

18. Bludau S, Eickhoff SB, Mohlberg $\mathrm{H}$, et al. 
Cytoarchitecture, probability maps and functions of the human frontal pole. Neuroimage 93(1), 260-275 (2014).

19. Bludau S, Bzdok D, Gruber O, et al. Medial prefrontal aberrations in major depressive disorder revealed by cytoarchitectonically informed voxel-based morphometry. Am. J. Psychiatry 173(3), 291-298 (2016).

20. Veer I, van der Wee N, Beckmann C, et al. Whole brain resting-state analysis reveals decreased connectivity in major depressive disorder. Eur. Neuropsychopharmacol 19(1), S399-S400 (2009).

21. Wang J, Wei Q, Bai T, et al. Electroconvulsive therapy selectively enhanced feedforward connectivity from fusiform face area to amygdala in major depressive disorder. Soc. Cogn. Affect. Neurosci (2017).

22. Hamilton M. A rating scale for depression. J. Neuro. Neurosurg. Psych 23(1), 56-62 (1960).

23. Burgess PW, Dumontheil I, Gilbert SJ. The gateway hypothesis of rostral prefrontal cortex (area 10) function. Trends. Cogn. Sci 11(7), 290-298 (2007).

24. Gilbert SJ, Gonen-Yaacovi G, Benoit RG, et al. Distinct functional connectivity associated with lateral versus medial rostral prefrontal cortex: A meta-analysis. Neuroimage 53(4), 1359-1367 (2010).

25. Farah MJ, Aguirre GK. Imaging visual recognition: Pet and fmri studies of the functional anatomy of human visual recognition. Trends. Cogn. Sci 3(5), 179-186 (1999).

26. McCarthy G, Puce A, Gore JC, et al. Facespecific processing in the human fusiform gyrus. J. Cogn. Neurosci 9(5), 605-610 (1997).

27. Staffaroni AM, Melrose RJ, Leskin LP, et al. The functional neuroanatomy of verbal memory in Alzheimer's disease: [18f]fluoro-2-deoxy-d-glucose positron emission tomography (fdg-pet) correlates of recency and recognition memory. J. Clin. Exp. Neuropsychol 1-13 (2016).

28. Bergmann HC, Daselaar SM, Fernandez G, et al. Neural substrates of successful working memory and long-term memory formation in a relational spatial memory task. Cogn. Process 17(4), 377-387 (2016).

29. Bergmann HC, Rijpkema M, Fernandez G, et al. Distinct neural correlates of associative working memory and long-term memory encoding in the medial temporal lobe. Neuroimage 63(2), 989-997 (2012).
30. Kim H, Cabeza R. Differential contributions of prefrontal, medial temporal, and sensory-perceptual regions to true and false memory formation. Cereb. Cortex 17(9), 2143-2150 (2007).

31. Liu F, Hu MR, Wang SS, et al. Abnormal regional spontaneous neural activity in first-episode, treatment-naive patients with late-life depression: A resting-state fmri study. Prog. Neuro-Psychopharmacol. Biol. Psych 39(2), 326-331 (2012).

32. Tomasi D, Caparelli EC, Chang L, et al. Fmriacoustic noise alters brain activation during working memory tasks. Neuroimage $27(2)$ 377-386 (2005)

33. Stoodley CJ, Valera EM, Schmahmann JD. An fmri study of intra-individual functional topography in the human cerebellum. Behav. Neurol 23(1-2), 65-79 (2010).

34. Peng J, Liu JT, Nie BB, et al. Cerebral and cerebellar gray matter reduction in firstepisode patients with major depressive disorder: A voxel-based morphometry study. Eur. J. Radiol 80(2), 395-399 (2011).

35. Guo WB, Sun XL, Liu L, et al. Disrupted regional homogeneity in treatmentresistant depression: A resting-state fmri study. Prog. Neuro-Psychopharmacol. Biol. Psych 35(5), 1297-1302 (2011).

36. Liu F, Guo W, Liu L, et al. Abnormal amplitude low-frequency oscillations in medication-naive, first-episode patients with major depressive disorder: A restingstate fmri study. J. Affect. Disord 146(3), 401 406 (2013).

37. Shelton RC, Sanders-Bush E, Manier $\mathrm{DH}$, et al. Elevated 5-HT 2a receptors in postmortem prefrontal cortex in major depression is associated with reduced activity of protein kinase a. Neuroscience 158(4), 1406-1415 (2009).

38. Bench CJ, Friston KJ, Brown RG, et al. Regional cerebral blood-flow in depression measured by positron emission tomography - the relationship with clinical dimensions. Psychol. Med 23(3), 579-590 (1993).

39. Liu CH, Ma X, Wu X, et al. Resting-state brain activity in major depressive disorder patients and their siblings. J. Affect. Disord 149(1-3), 299-306 (2013).

40. Liu Z, Guo H, Cao X, et al. A combined study of gsk3beta polymorphisms and brain network topological metrics in major depressive disorder. Psychiatry. Res 223(3), 210-217 (2014).

41. Peng W, Chen ZQ, Yin L, et al. Essential brain structural alterations in major depressive disorder: A voxel-wise meta-analysis on first episode, medication-naive patients. J. Affect. Disord 199(1), 114-123 (2016).

42. Qiu L, Lui S, Kuang W, et al. Regional increases of cortical thickness in untreated, first-episode major depressive disorder. Transl. Psych 4 (2014).

43. Lener MS, Kundu P, Wong E, et al. Cortical abnormalities and association with symptom dimensions across the depressive spectrum. J. Affect. Disord 190(1), 529-536 (2016).

44. Hermesdorf M, Sundermann B, Feder S, et al. Major depressive disorder: Findings of reduced homotopic connectivity and investigation of underlying structural mechanisms. Hum. Brain. Map 37(3), 12091217 (2016).

45. Liu F, Guo WB, Liu L, et al. Abnormal amplitude low-frequency oscillations in medication-naive, first-episode patients with major depressive disorder: A restingstate fmri study. J. Affect. Disord 146(3), 401406 (2013).

46. Haldane M, Cunningham G, Androutsos $C$, et al. Structural brain correlates of response inhibition in bipolar disorder. J. Psychopharmacol 22(2), 138-143 (2008).

47. Chantiluke K, Halari R, Simic M, et al. Fronto-striato-cerebellar dysregulation in adolescents with depression during motivated attention. Eur. Neuropsychopharmacol 22(1), S84-S84 (2012)

48. Hach S, Tippett LJ, Addis DR. Neural changes associated with the generation of specific past and future events in depression. Neuropsychologia 65(1), 41-55 (2014).

49. Semkovska M, McLoughlin DM. Objective cognitive performance associated with electroconvulsive therapy for depression: A systematic review and meta-analysis. Biol. Psych 68(6), 568-577 (2010).

1. McCabe C, Mishor Z. Antidepressant medications reduce subcortical-cortical resting-state functional connectivity in healthy volunteers. Neuroimage 57(4), 1317-1323 (2011). 